\title{
Plant Growth Promoting (PGP) Activities of Rhizobial Isolates from Sesbania bispinosa in Response to Pesticides
}

\author{
Moumita Chakrabarty, Farhad Hossen, Anowara Begum and Humaira Akhter* \\ Department of Microbiology, University of Dhaka
}

\begin{abstract}
The aim of this study was to investigate the impact of some pesticides on the plant growth promoting activities of Rhizobium spp. isolated from Sesbania bispinosa. Sustainability and environmental safety of agricultural production relies on eco-friendly approaches like use of biofertilizers, biopesticides and crop residue return. The plant growth promoters (PGP) emphasize the need for further strengthening the research and their use in modern agriculture. PGPs inhabit the rhizosphere for nutrients from plant root exudates. On return, they help to increase plant growth through soil nutrient enrichment by nitrogen fixation, phosphate solubilization, siderophore and phytohormone production, etc. Using pesticide/insecticide in the field may protect the plants from undesirable organisms, however, if accumulated in the habitat may adversely affect the growth and development of non-target lives like the rhizobacteria. The present study was performed to demonstrate the influence of pesticides on plant growth promoting activities of rhizobial isolates from Sesbania bispinosa known as green manure among farmers of Bangladesh. It was observed that two pesticides/insecticides (Imitaf 20SL and Tilt 250EC) in vitro shows variable effect on the plant growth promoting activities of the rhizobial isolates.
\end{abstract}

Key Words: Plant growth promoter (PGP), Pesticide, Rhizobium, Sesbania bispinosa.

\section{Introduction}

When a pesticide is introduced into agricultural practice for the purpose of plant protection, it is necessary to investigate whether the soil microflora and the biological processes in the soil will be affected, particularly when there is even a small chance of the chemicals reaching the soil. There are several possible detrimental effects of the pesticides on encountering the soil. One important possibility is a direct action on soil microorganisms consequently disturbing many fundamental biochemical processes. Soil fertility is related to a very delicate equilibrium and its disturbance may affect fertility in a wrong way. Considering this, it is especially important to know the behavior of pesticides in the soil and it is not surprising that considerable numbers of studies have been made on the effect of pesticides on biological processes or on the behavior of microbial populations in soil.

Sesbania is a genus of approximately 60 species of tropical legumes of which 40 have so far been reported to nodulate ${ }^{1}$. Many species occur naturally in wet or flooded soils, and these have considerable potential as green manure in wetland rice production due to their ability to fix large quantities of $\mathrm{N}_{2}{ }^{2}$. Sesbania nodules may be induced by a variety of rhizobia, including Azorhizobium spp. ${ }^{3}$, Mesorhizobium spp. and Rhizobium spp. Producers can use seeds of sesbania to add rhizobia to the soil to ensure that their legume crops have a source of $\mathrm{N}_{2}$ by adding appropriate species of Rhizobium to the seeds of crop. The resultant Rhizobia-Legume symbiosis can benefit not only the host, but it may also have positive effects for the subsequent crops by adding nitrogenous residues to the soil.

Rhizobia is the common name given to a group of small, rodshaped, Gram-negative bacteria that collectively can produce nodules on the roots of leguminous plants and belong to the family Rhizobiaceae, which are part of the proteobacteria. Rhizobium, Sinorhizobium, Mesorhizobium and Bradyrhizobium, are also producers of different extra ploysaccharides from simple glycans to complex heteropolymers ${ }^{4}$. Rhizobia are the most studied PGPR (Plant growth promoting rhizobacteria) for their potential to fix $\mathrm{N}_{2}$ in the leguminous plants. The symbiosis between a leguminous plant and its microsymbiont is characterized by a multistep signal exchange process. During growth in the rhizosphere of a host plant, rhizobia sense compounds such as flavonoids and betanoides secreted by the host root and respond by inducing nod genes ${ }^{5}$.

The Rhizobium-legume symbiosis and its different aspects were studied from time to time. The interest of researchers has resulted in the elucidation of several aspects of the physiology of Rhizobium-legume symbiosis. A successful symbiosis is the result of a complex series of interactions between the host and the symbiont. To form an effective symbiosis, rhizobia require several classes of specific genes. These include nod genes, encoding the production of Nod factors which stimulate the plants to produce symbiotic nodules, and nif genes which produce the nitrogenfixing nitrogenase enzyme ${ }^{6}$. 
Although uses of pesticides is effective for the protection of plants from pest, the extensive use of these over the past few decades has resulted in the disturbance of natural biological system. Some pesticides used in agriculture can have direct harmful affect to nitrogen fixing bacteria. The objective of the present study was to study the effect of pesticides on the PGP activities of nitrogen fixing Rhizobium isolates from Sesbania bispinosa.

The present study assessed the activities of the plant growth promoting rhizobial isolates under the effect of different pesticides. For this purpose, two different pesticides namely Imitaf 20SL and Tilt 250EC were used at various concentrations ${ }^{7}$. Imitaf 20SL is one of the neonicotinoid type pesticides. Imidacloprid is the active ingredient of Imitaf which is a systemic insecticide acting as an insect neurotoxin. Tilt 250EC is a fungicide having propiconazole as its active ingredient. It achieves a high-level control in spraying programs contributing significantly to avoiding yield and quality losses.

\section{Materials and Methods}

In this study, 20 isolates were investigated which were collected from the root nodules of Sesbania bispinosa by a previous worker ${ }^{7}$ and were grown on $\mathrm{N}_{2}$-free medium (Jensen media). Different plant growth promoting activities viz. ammonia production, phosphate solubilization and indole acetic acid (IAA) production $^{8}$ of the rhizobial isolates were observed with and without the presence of pesticides in the growth media. The antibiotic sensitivities of the isolates were assessed under the influences of pesticides in the growth media. The isolates were tested against two different concentrations of pesticides (Imitaf 20SL and Tilt 250EC). Since all isolates showed maximum growth at $0.5 \%$ pesticide, all tests were performed in the presence of $0.5 \%$ pesticides.

\section{Growth on N-Free Media}

The nitrogen fixation ability of the isolates was evaluated by growing them on $\mathrm{N}$-free Jensen's ${ }^{9}$ medium along with different pesticides. The isolates were streaked on semisolid $-\mathrm{JNFb} /$ LGI(Jensen's Nitrogen-Fixing bacteria medium/Liquid Glucose Ivo) medium and incubated at $28^{\circ} \mathrm{C}$ for 24 to 72 hours. Observation of the bacterial growth on the plates was considered as qualitative evidence of atmospheric nitrogen fixation ${ }^{10}$.

\section{Ammonia Production Assay}

Each isolate was inoculated into $5 \mathrm{ml}$ peptone medium along with each type of pesticides and incubated for $48 \mathrm{~h}$ at $30^{\circ} \mathrm{C}$. After the bacterial growth, Nessler's reagent $(0.5 \mathrm{ml})$ was added to each tube in 2:1 ratio. Development of brown to yellow color was considered as positive test for ammonia production. Uninoculated medium was used as reference.

\section{Antibiotic Sensitivity Test under the Effect of Pesticides}

Bacterial suspensions were prepared in Muller-Hinton broth. After incubation, the turbidity of the broth was adjusted to 0.5 McFarland standards. A fresh, sterile cotton swab was dipped into the suspension and a lawn of the tested organism was prepared on Muller-Hinton agar medium. Excess liquid was removed from the swab by pressing it against the side of the tube before making the lawn. Antimicrobial discs (Neomycin, Sulphomethaxozole trimethoprim and chloramphenicol) were applied on the plates by placing with the help of sterile forceps. After incubation, the zone of inhibition formed was measured in $\mathrm{mm}^{7}$.

\section{Phosphate Solubilization Efficiency}

Pure cultures of bacteria were spot inoculated at the center of prepared plates of NBRIP (National Botanical Research Institute's phosphate growth medium) agar medium with and without pesticides ${ }^{7}$. The plates were incubated at $30 \pm 2^{\circ} \mathrm{C}$ for 7 days. Isolates that were capable of solubilizing phosphate formed a clear zone around the colony and were considered as positive for phosphate solubilization. The zone of phosphate solubilization $(\mathrm{cm})$ formed around the colonies were recorded after 7 days of incubation. On the other hand, absence of clear zone around the colony indicated no phosphate solubilization. After incubation, the zone of phosphate solubilization and colony diameter were measured. Then, phosphate solubilizing efficiency of the microorganisms was calculated using the following formula:

Solubilization Index $=\frac{\mathrm{Z}+\mathrm{c}}{\mathrm{c}}$, where $\mathrm{Z}=$ Solubilization zone $(\mathrm{cm})$ and $\mathrm{C}=$ Colony diameter $(\mathrm{cm}){ }^{7}$

\section{Quantitative IAA production assay}

IAA production assay was conducted by following the method of Gordon \& Weber ${ }^{8}$. Yeast extract-malt extract-dextrose agar media (YMD) was inoculated with bacteria with $0.1 \%$ tryptophan along with different types of pesticides. Autoclaved uninoculated YMD media containing test tubes were taken as controls. The tubes were incubated for 5 days at $30^{\circ} \mathrm{C}$ at $120 \mathrm{rpm}$ in shaker incubator. After incubation, the broths were centrifuged. IAA production level was determined with Salkowski reagent. Two $\mathrm{ml}$ of Salkowski reagent was added with $1 \mathrm{ml}$ incubated medium and left in the dark for 30 minutes at room temperature. The optical density was measured at $530 \mathrm{~nm}$. Two readings for optical density were taken and the mean absorbance value was calculated. Using the standard curve for IAA, the amount of IAA produced by each sample was calculated ${ }^{7}$.

\section{Results}

Growth on $\mathrm{N}_{2}$-free medium

All isolates exhibited growth both in the presence and absence of pesticides on $\mathrm{N}_{2}$-free (Jensen's) medium. Uninoculated medium was taken as the references where no growth was observed.

\section{Ammonia production test}

All isolates produced ammonia both in the presence and absence of the pesticides. Development of brown to yellow color was observed as positive test for ammonia production at both 0.25 and $0.5 \%$ pesticides. Uninoculated medium was used as the reference which did not show any change in color. 


\section{Antibiotic Sensitivity test}

More than $70 \%(n=15)$ isolates showed sensitive towards the three antibiotics tested both in the presence and absence of added pesticides. The rest showed intermediate resistance in the presence/absence of pesticides.

\section{Effect of Pesticides on Phosphate solubilization}

Comparison of phosphate solubilization (with and without pesticide)

The isolates exhibited higher phosphate solubilization efficiency in the presence of $0.5 \%$ Tilt in comparison to similar concentration Imitaf as documented in Fig. 1. Isolates growing in the absence of pesticides showed less ability for solubilizing phosphate in the experiment (Fig. 1).

Effect of two Different Concentration of Tilt 250EC on Phosphate Solubilization

All the isolates exhibited higher phosphate solubilization in the presence of tilt than in the absence of pesticide. It was observed that, with the increased concentration of Tilt $250 E C$, the phosphate solubilization increased as well (Fig. 2).

Effect of different concentration of Imitaf 20SL On Phosphate Solubilization

From Fig. 3 it could be concluded that isolates exhibited higher phosphate solubilization in the presence of Imitaf 20SL than in the absence of it. With the increase of pesticide concentration from $0.25 \%$ to $0.5 \%$, the phosphate solubilization increased in most of the isolates.

\section{Effect of pesticides on indole acetic acid production}

Comparison of IAA production (with and without pesticide)

All of the isolates exhibited higher production of IAA in the presence of $0.5 \%$ Tilt $250 \mathrm{EC}$ when compared with $0.5 \%$ Imitaf 25SL. IAA production was found to be higher even in the absence of pesticide. (Fig. 4). In some samples, IAA production without pesticide was higher than that of Tilt 250EC added samples.

Effect of two different concentrations of Tilt 250EC on IAA production In the presence of Tilt 250EC, the IAA production increased for most of the strains and other than a few cases, the production became more increased with the increment of its concentration (Fig. 5)

Effect of two different concentrations of Imitaf 20SL on IAA production It was observed that, in the presence of Imitaf 20SL, the isolates produced less Indole acetic acid (IAA) than in the absence of it. Furthermore, it was observed that with the increased concentration of Imitaf 20SL, the IAA Production became even lesser (Fig. 6).

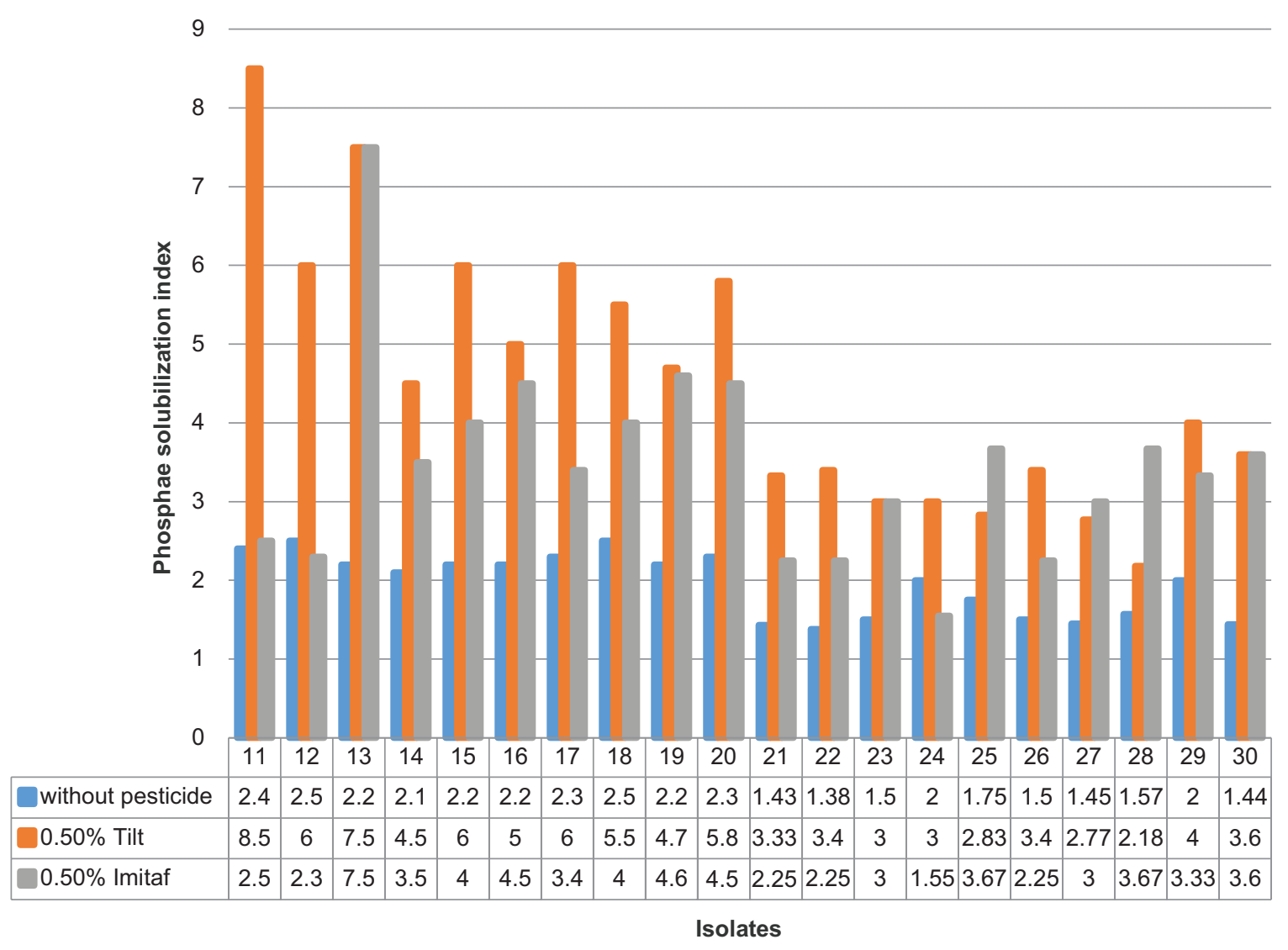

Fig. 1. Variation in phosphate solubilization between Imitaf250SL and Tilt25EC pesticides. 


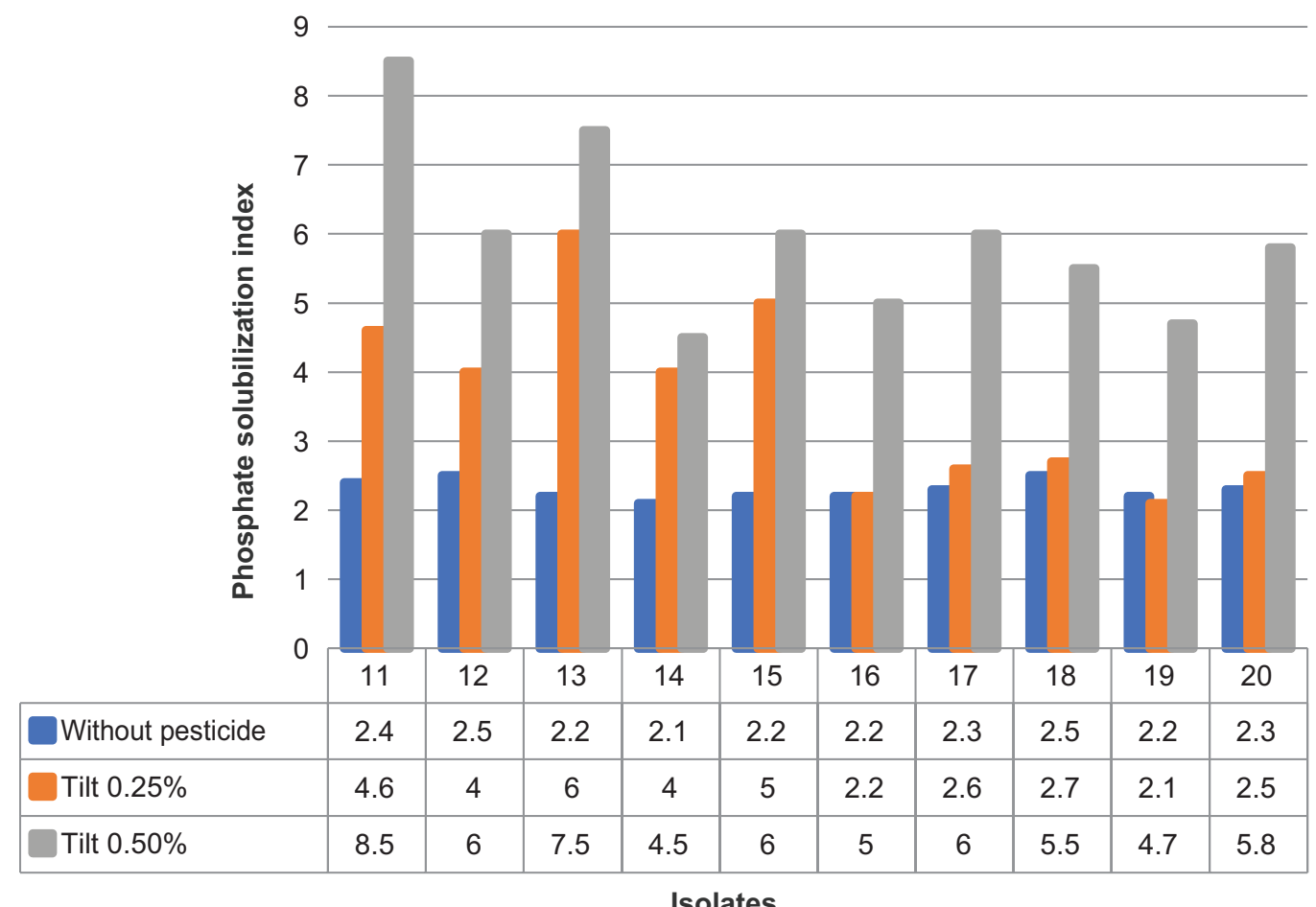

Isolates

Fig. 2. Effect of two different concentrations of Tilt 250EC on phosphate solubilization.

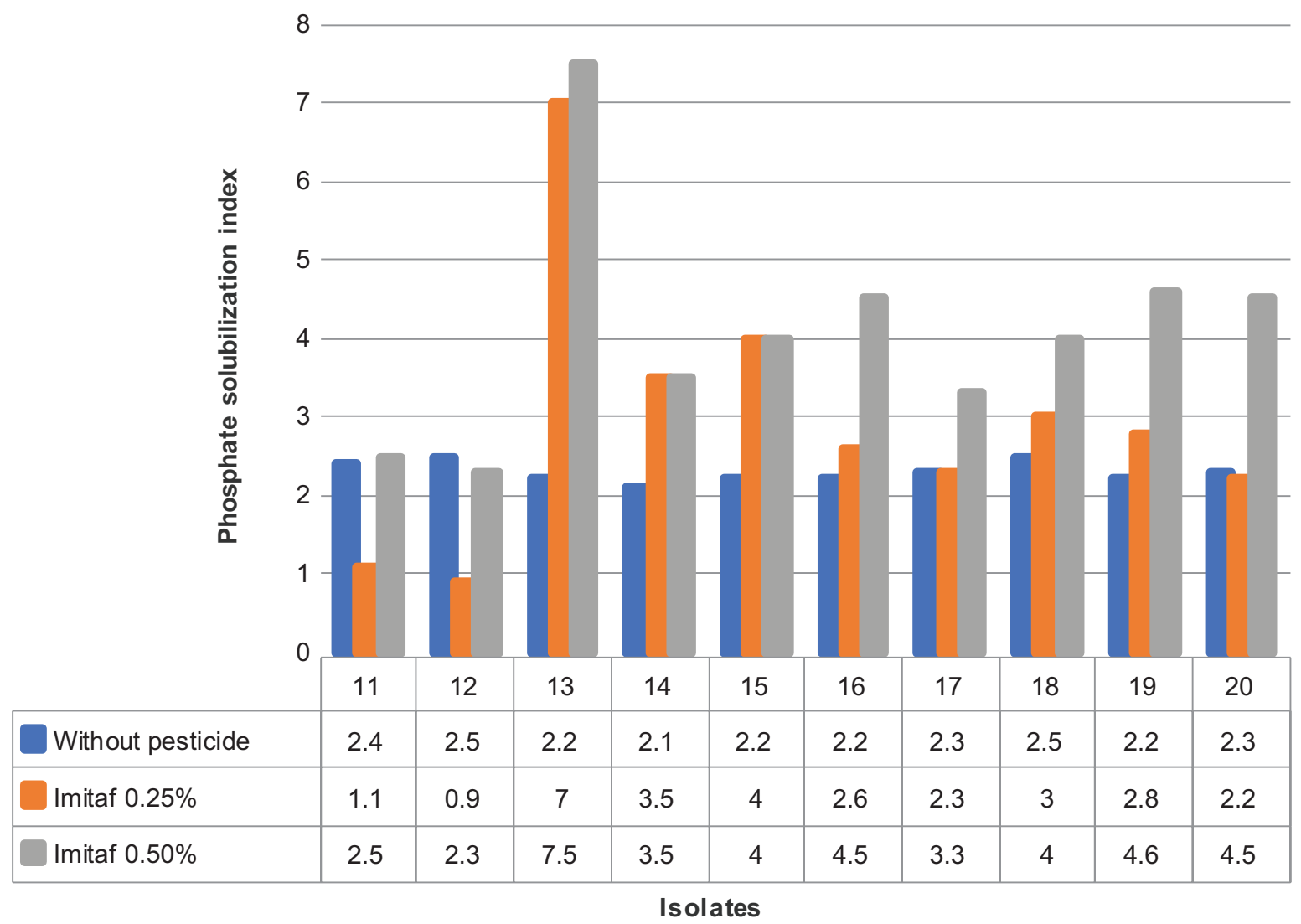

Fig 3. Effect of two different concentrations of Imitaf 20SL on phosphate solubilization. 


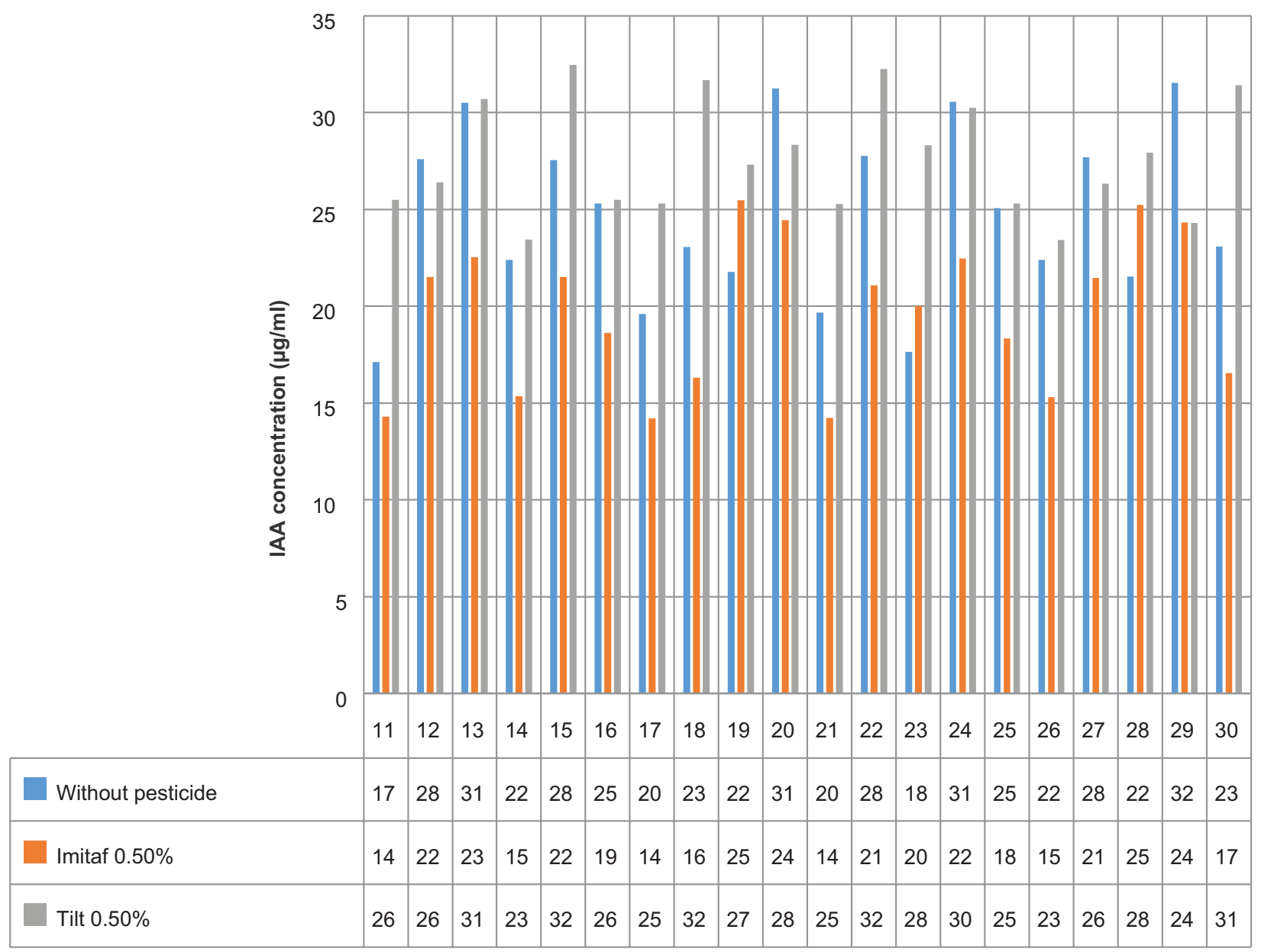

Isolates

Fig. 4. Variation in IAA production with $0.5 \%$ Tilt 250EC, $0.5 \%$ Imitaf $25 S L$ and without pesticide.

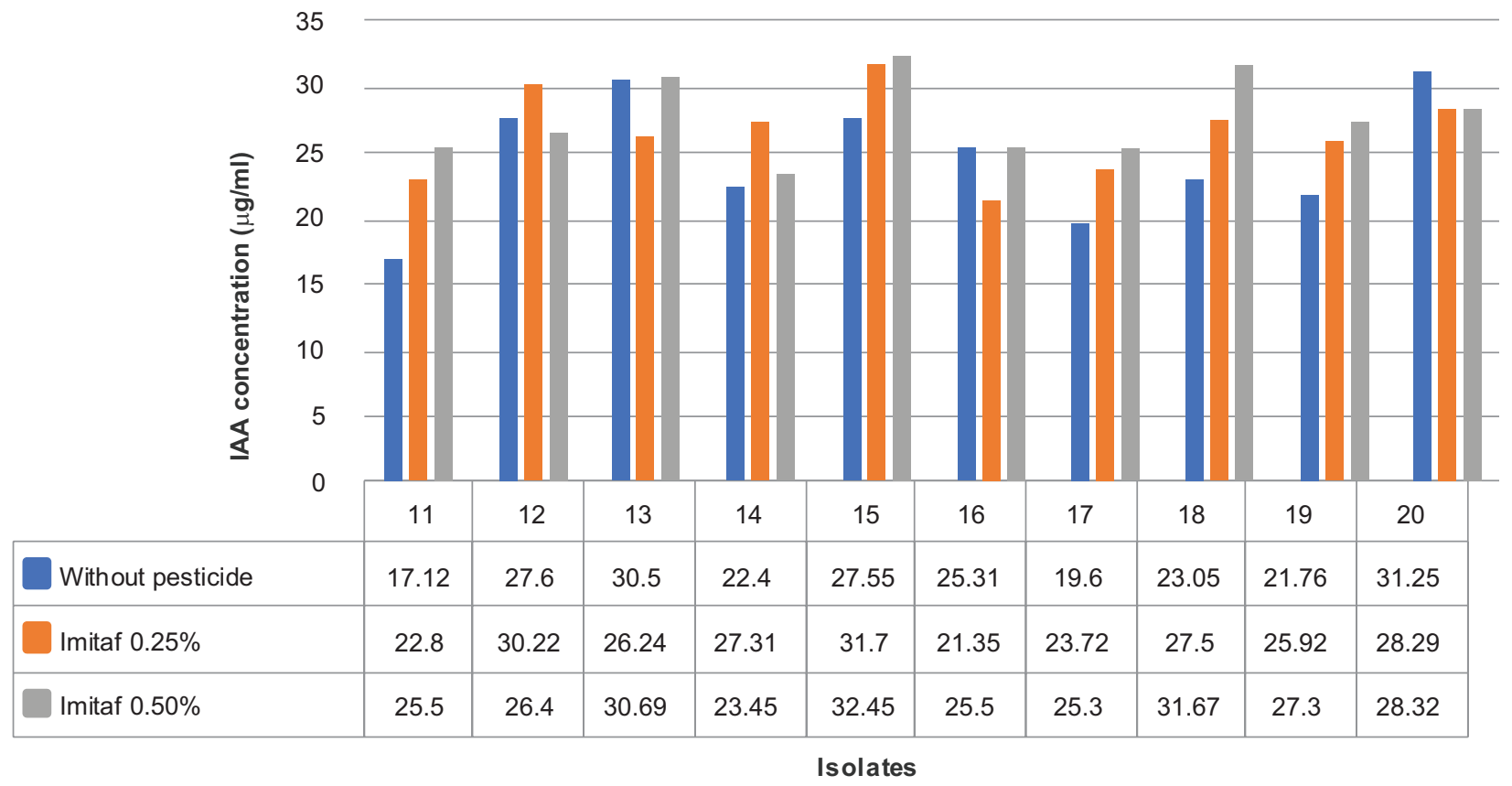

Fig. 5. IAA production at two different concentrations of Tilt 250EC. 


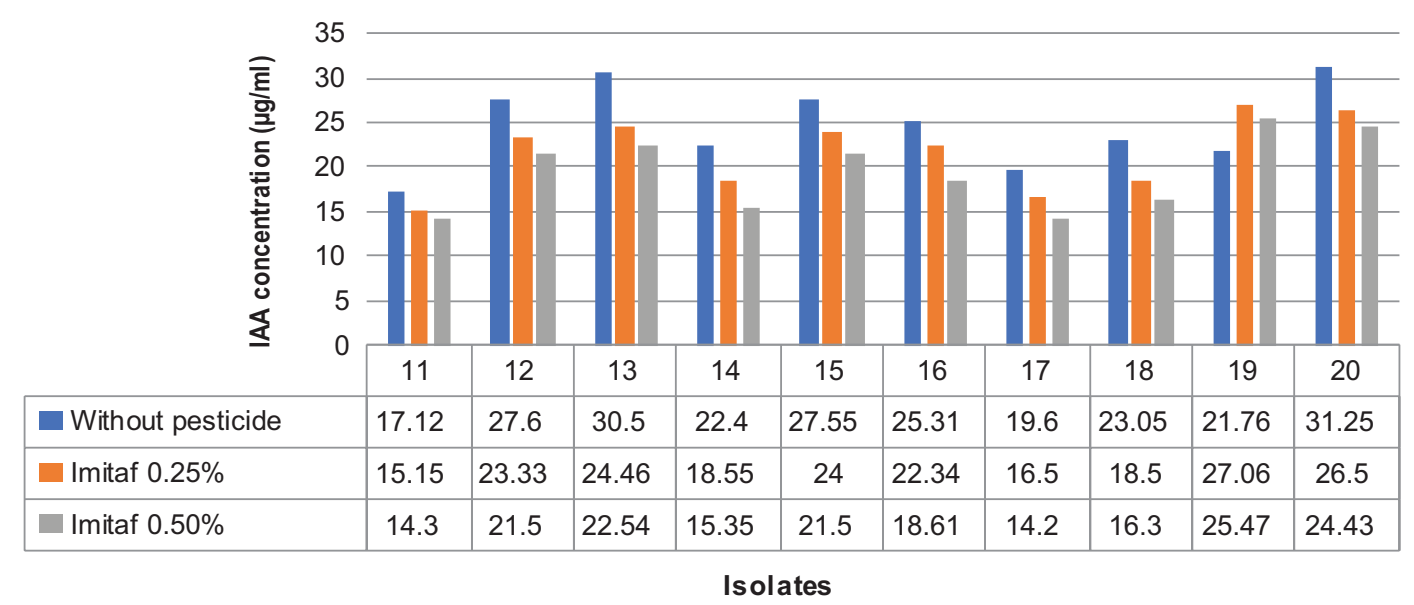

Fig. 6. IAA production at two different concentrations of Imitaf 20SL.

\section{Discussion}

The relationship of nitrogen-fixing bacteria with roots of higher plants has long been an intricate part of agriculture. Farmers in poorer countries especially cannot afford to lose natural fertilizer because of the high cost of artificial. The rhizosphere of plants is a zone of intense microbial activities that exerts beneficial effects on plants. These plant growth promoters (PGP) if they are affected negatively, will inhibit growth and yield of plants.

The present research project was carried out for observing the effect of pesticides namely Imitaf 25SL and Tilt 250EC on desired plant growth promoting activities of Rhizobium spp. whose activities are essential for the growth and yields of plants. All the isolates exhibited growth on $\mathrm{N}_{2}$-free media in presence of different pesticides, which indicated the qualitative evidence of atmospheric nitrogen fixation. All the isolates could produce ammonia and could solubilize phosphate, which was found to be efficiently increased in the presence of pesticides. Most of the rhizobial isolates were found to be sensitive to antibiotics both in the presence and absence of pesticides. IAA production was reduced with the pesticide Imitaf $25 \mathrm{SL}$ in comparison to that (Fig. 6) where it was increased in the presence of Tilt 250EC (Fig. 5).

Generally, the use of pesticides is supposed to be good for crops. Results of the present study concluded as was previously done, that the two pesticides used have differential effects on the growth of the microorganisms and on the plant growth promoting activities of the nitrogen fixing bacteria ${ }^{7}$.

In one study, it was found that numerous microorganisms have the capacity to degrade the pesticides by the action of degradative genes carried in plasmids, transposons or chromosomes ${ }^{10}$. Pesticides vary in their toxicology to organisms owing to their functional groups and a great degree of variability occurs even among pesticides of similar functional groups ${ }^{11}$. Pesticides are widely used by producers to obtain maximum quality and yield. However, all classes of these chemicals contain compounds which have reduced nodulation and/or nitrogen fixation in free-living organisms and in symbionts ${ }^{12}$.

Due to the indiscriminate use of pesticides, the issue of the impact of these chemicals on the environment and soil has gained attention. Only about $0.1 \%$ of the total applied pesticides reach the target site/organism, while the remaining bulk contaminates the soil environment ${ }^{13}$. Consequently, sizable amounts of pesticides reach soil directly and indirectly, which affects the composition of soil microflora ${ }^{14}$. One of the most important and potentially limiting factors to biological nitrogen fixation is the use of chemical pesticides. Recommended doses of pesticides, in general, do not have any harmful effect on nitrogen fixation. At higher doses, various chemical pesticides affect the physiological and growth characteristics of both rhizobia and legume plants. They also inhibit the symbiotic nitrogen fixation by blocking biochemical communication and dialogues between the legumes and rhizobial symbionts ${ }^{15}$.

Phosphorus deficiency in soils affects nodule functioning; however, it does not inhibit the other aspects of plant growth and metabolism. Legume tissues do not appear to have higher $\mathrm{P}$ content than those of other plants. Therefore, a positive interaction exists between legume's growth and $\mathrm{N}$ fixation in response to $\mathrm{P}$ supply. As such, rhizobia with phosphate solubilization capacity can greatly influence nitrogen fixation in legume plants. In accordance with the findings of the present study, another study found increased phosphate in the presence of pesticides ${ }^{16,17}$. Initially though it seems that increasing phosphorous solubilization by rhizobacterium is beneficial for plants however it is not known what the pesticide concentration might affect in the long run.

One of the direct mechanisms by which PGPs promote plant growth is by production of plant growth regulators or phytohormones like Indole acetic acid (IAA) among others ${ }^{18}$. From the present study, it was found that the plant growth promoting activities of the rhizobial isolates varied with the type and concentration of pesticides. Furthermore, the production of 
phytohormones by the isolates suggested the potential of the plant growth promoting rhizobacteria to be used as biofertilizers. But further tests should be done to see how long the PGP activities can continue in presence of pesticiides /insecticides.

It should be mentioned that the effect of pesticides on soil microorganisms and on microbial transformations in soil largely depends on the dose and chemical structure of the preparations as well as on the available soil surface physical, chemical and biological soil conditions, and the presence of nitrogenous fertilizers. Further studies should be performed to see the effect of pesticides at different concentrations on other properties like siderophore production.

In conclusion, considering all the impacts of pesticides, awareness about the usage of pesticides must be raised to preserve both the environment and living organisms.

\section{Acknowledgement}

The authors express gratitude to the Ministry of Education, Government of the Peoples' Republic of Bangladesh for their support in conducting the project.

\section{References}

1. Sprent JI. 2001. Nodulation in legumes. London: Royal Botanic Gardens, Kew. Penny Hirsh, London.

2. Lennox JG. 2001. Aristotle's philosophy on biology studies on the origins of life science. Cambridge University press.

3. Azevedo JLD and Quecine MC. Diversity and Benefits of Microorganisms from the Tropics. Springer, Cham.

4. Monteiro I and Nilson K. 2012. Chemical characterization of the gels produced by the diazotrophic bacteria Rhizobium tropici and Mesorhizobium sp. Química Nova. 35: 705-708.

5. Skorupska. A., Janczarek. M., Marczak. M., Mazur, A., and Krol, J. 2006. Rhizobial exopolysaccharides: genetic control and symbiotic functions. Microbial Cell Factories 2006, 5:(7) DOI:10.1186/1475-2859-5-7.

6. Haukka K, Lindstrom K and Young JPW. 1998. Three phylogenetic groups of nod $A$ and nif $H$ genes in Sinorhizobium and Mesorhizobium isolates from leguminus trees growing in Africa and Latin America. Appl Environ Microbiol. 64: 419-426.

7. Sultana T, Begum A and Akhter H. 2019. Effect of pesticides on Exopolysaccharide (EPS) production, antibiotic sensitivity, and phosphate solubilization by Rhizobial isolates from Sesbania bispinosa in Bangladesh. African Journal of Agricultural Research 14(34): 1833-1844 DOI:10.5897/AJAR2019.14304

8. Gordon SA and Weber RP. 1951. Colorimetric estimation of indole acetic acid. Plant Physiol. 26: 192-195. (DOI: 10.1104/pp.26.1.192).

9. Jensen HL, 1942. Nitrogen fixation in leguminous plants. II. Is symbiotic nitrogen fixation influenced by Azotobacter? Pro Line Soc. N.S.W., 57: pp:205-212.

10. Doebereiner J. 1995. Isolation and identification of aerobic nitrogen fixing bacteria from soil and plants. Methods in applied soil microbiology and biochemistry Academic, London, 134-141.

11. Kumar S, Mukerji KG and Lal R.1996. Molecular aspects of pesticide degradation by microorganisms.. Crit Rev Microbiol. 22: 1-26.

12. Ahemad M and Khan MS. 2011. Pesticide interactions with soil microflora: importance in bioremediation. In: Ahmad I, Ahmad F, Pichtel J. (Eds.), Microbes and Microbial Technology: Agricultural and Environmental Applications. Springer, New York, pp. 393-413.

13. Moiroud A and Faure-Raynaud M. 1983. Effects of some herbicides on the growth and infectivity of Frankia strains. Plant and Soil. 74: 133-136.

14. Carriger JF, Rand GM, Gardinali PR, Perry WB, Tompkins MS and Fernandez AM. 2006. Pesticides of potential ecological concern in sediment from South Florida Canals: An ecological risk prioritization for aquatic arthropods. Soil and Sediment Contamination. 15: 21-45.

15. Andrea MM, Peres TB, Luchini LC and Pettinelli A. 2000. Impact of long-term pesticide application on some soil biological parameters. Journal of Environmental Science and Health, Part B. 35(3): 297-307.

16. Ahemad M and Khan MS. 2013. Pesticides as antagonists of rhizobia and the Legume- Rhizobium Symbiosis: A paradigmatic and mechanistic outlook. Biochemistry and Molecular Biology. 1(4): 63-75.

17. Ramani V. 2011. Effect of pesticides on phosphate solubilization by Bacillus sphaericus and Pseudomonas cepacian. Pesticide Biochemistry and Physiology. 99: 232-236.

18. Glick BR. 1995. The enhancement of plant growth by free living bacteria. Can. J. Microbiol. 41: 109-117. 